http://dx.doi.org/10.22319/rmcp.v9i2.4478

Artículo

\title{
Comportamiento epidemiológico de Cystoisospora suis en granjas porcinas ubicadas en la región central de Venezuela
}

\section{Epidemiological aspects of Cystoisospora suis in swine herds located at the} Central region of Venezuela

Juan Carlos Pinilla León ${ }^{\text {ab * }}$

Natalia Da Silva Borges ${ }^{b}$

${ }^{a}$ Universidad de Santander, Facultad de Ciencias Agropecuarias, Programa de Medicina Veterinaria, Campus de Bucaramanga, Lagos de Cacique, Bucaramanga, Santander, Colombia.

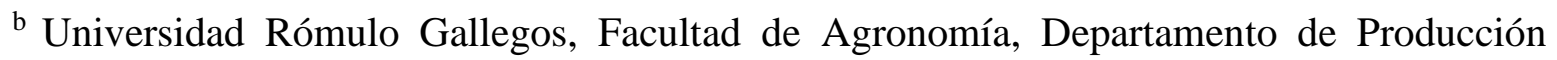
Animal, San Juan de Los Morros, Venezuela.

* Autor de correspondencia: j.pinilla@mail.udes.edu.co

\section{- Resumen:}

Se condujo una investigación en Venezuela durante el año 2016 con el propósito de estudiar el comportamiento epidemiológico de Cystoisospora suis. Se incluyeron 67 granjas porcinas intensivas con antecedentes de diarrea neonatal. Para la determinación parasitaria se seleccionaron 572 camadas, así como 1,712 muestras fecales de cerdos adultos. En todas las granjas se aplicó una encuesta epidemiológica escrita. Todas las muestras se cultivaron en dicromato de potasio al $2.5 \%$ y posteriormente se procesaron con una técnica coproparasitológica. Los resultados señalan que $C$. suis se encontró en 55 de 67 granjas (82.1\%) y 210 camadas $(36.7 \%)$, con los mayores valores de frecuencia en las dos primeras semanas de vida $(P<0.05)$. Al referir los resultados en cerdos adultos se encontró correlación significativa $(\mathrm{rho}=0.35 ; P<0.05)$ entre infección de lechones y cerdas, lo que sugiere que cerdas madres podrían actuar como fuentes de infección. El número de partos se encontró 
estadísticamente correlacionado con los valores de frecuencia en camadas y cerdas $(P<0.05)$, lo que indica que, a mayor número de partos, disminuye la frecuencia. Probablemente estos hallazgos se asocian con mecanismos inmunológicos desconocidos. La infección en camadas menores a tres días de edad, presupone la existencia de alguna ruta alternativa de la infección. Se concluye que $C$. suis se encuentra ampliamente distribuida en la región central de Venezuela y que pudiera ser controlada mejorando las condiciones sanitarias de las granjas; sin embargo, mecanismos inmunológicos aún sin dilucidar podrían estar involucrados en la transmisión del protozoario.

- Palabras clave: Cystoisospora suis, Epidemiológico, Granjas, Porcinos.

\section{- Abstract:}

It was carried out an investigation during 2016 with the aim to study epidemiological aspects of Cystoisospora suis infections. Sixty-seven (67) intensive swine herds with a history of neonatal diarrhea were included. Overall, 572 litters and 1,712 fecal samples in mature pigs were examined. A written epidemiological survey was applied on all farms. Fecal samples were cultured in $2.5 \%$ potassium dichromate and analyzed with a copro-parasitological technique. C. suis was found in 55/67 herds $(82.1 \%)$ and 210 litters $(36.7 \%)$ with highest prevalence in the first $2 \mathrm{wk}$ of age $(P<0.05)$. Regarding mature pigs, a significant correlation was found (rho $=0.35 ; P<0.05)$ between infection in piglets and sows, suggesting that mothers sows could act as infection sources. Statistical associations were determined among parity number and frequency values in litters and sows $(P<0.05)$, indicating that as parity increase, prevalence decrease. Probably these findings are associated with unknown immunologic mechanisms. Infection in litters less than $3 \mathrm{~d}$ of age, presupposes the existence of some alternative infection route. It is concluded that $C$. suis is broadly distributed at the central region of Venezuela and it could be controlled by improving the sanitary conditions of the herds; however, immunologic mechanisms might be involved in the protozoa transmission cycle which remain yet to be determined.

- Key words: Cystoisospora suis, Epidemiology, Herds, Swine.

Recibido el 02/05/17.

Aceptado el 03/10/17. 


\section{Introducción}

La cystoisosporosis porcina es una enfermedad causada por Cystoisospora suis, un protozoario con distribución cosmopolita, y que se encuentra en cerdos mantenidos en confinamiento. Los animales que se infectan desarrollan una diarrea amarillenta a partir de la segunda semana de edad, y su prevalencia es muy variada ${ }^{(1)}$. La epidemiología de $C$. suis aún es confusa y se piensa que las cerdas madres pueden jugar un papel importante en la transmisión; sin embargo, algunos investigadores no encontraron cerdas excretando ooquistes, concluyendo que el medio ambiente es la fuente de infección para los lechones ${ }^{(1,2)}$. Otros autores señalaron excreción de ooquistes de $C$. suis en cerdas madres $^{(3,4,5)}$, y en lechones destetados ${ }^{(6)}$, concluyendo que cerdas madres y lechones de iniciación podrían jugar un rol en la transmisión del parásito.

Con respecto a la prevalencia, en Australia, se determinó $53.8 \%$ de prevalencia en lechones lactantes diarreicos con edades entre 5 y 30 días $^{(7)}$, mientras que en EE.UU, señalaron que todas las granjas examinadas estaban contaminadas, con una prevalencia de $62.2 \%$ en lechones lactantes ${ }^{(8)}$. En Alemania, se reportó $42.5 \%$ de prevalencia en camadas ${ }^{(9)}$, mientras que en la república Checa se señaló $21.8 \%$ de prevalencia ${ }^{(10)}$. En Venezuela, se determinó $21.8 \%$ de prevalencia en lechones criados en granjas de los estados Aragua y Carabobo, posteriormente, demostraron $75 \%$ de prevalencia en granjas con manejos eficientes y deficientes, concluyendo que el parásito se presentó en cualquier tipo de explotación ${ }^{(11)}$.

El número de partos juega un rol importante sobre el sistema inmune de la cerda, ya que se ha demostrado disminución de anticuerpos contra ciertas infecciones ${ }^{(12,13)}$, sobre todo en primerizas, las cuales se pueden ver inmunocomprometidas por efecto del parto y $\operatorname{lactancia}^{(14,15)}$.

La región central de Venezuela cuenta con más del $70 \%$ del total de granjas intensivas del país y alrededor de 80 mil cerdas en producción, representando $53 \%$ del total de vientres en Venezuela, lo que hace esta región representativa de la producción porcícola nacional ${ }^{(16)}$. Por lo tanto se planteó como objetivo del presente trabajo determinar el comportamiento epidemiológico de Cystoisospora suis en granjas porcinas de producción intensiva de la región central de Venezuela.

\section{Material y métodos}




\section{- Ubicación geográfica del estudio}

Las granjas examinadas se encuentran localizadas en la región central de Venezuela, específicamente en los estados Aragua y Carabobo. Las características climatológicas de la región son consideradas como de clima tropical, con registros pluviométricos anuales entre 500 y $1,450 \mathrm{~mm}$, temperaturas medias anuales entre 24.5 y $27{ }^{\circ} \mathrm{C}$ con $70 \%$ de humedad relativa. Los estados están situados entre 600 y $2,400 \mathrm{msnm}^{(17)}$.

\section{- Selección de las granjas 。}

Se incluyeron únicamente explotaciones porcinas intensivas, con tamaño igual o mayor a 100 vientres en producción, lo que supone una sólida actividad económica de las mismas ${ }^{(18)}$. La mayoría de las granjas examinadas presentaban pobres condiciones higiénico - sanitarias y antecedentes de diarrea neonatal. El tipo de animal observado pertenece a mestizos de líneas mejoradoras, con alimentación a base de raciones balanceadas, las cuales son formuladas en plantas de alimentos próximas a las unidades de producción.

\section{- Diseño del muestreo}

La investigación realizada es de tipo descriptiva y de corte transversal. Para el estudio se diseñó un muestreo aleatorio en dos etapas por conglomerados ${ }^{(19)}$, empleando los registros de censos porcinos existentes en el Departamento de Epidemiología del Servicio Autónomo de Sanidad Agropecuaria ${ }^{(20)}$. En la primera etapa del muestreo, se seleccionaron $40 \%$ de los municipios de cada estado, los cuales se escogieron al azar utilizando una tabla de números aleatorios. En los municipios seleccionados se visitaron todas las granjas intensivas. Así, en Aragua se visitaron 43 granjas que representan el $60 \%$ del total de explotaciones intensivas 
del Estado, sin embargo, solamente se examinaron 32 granjas (73.6 \%), ya que al momento de la visita no permitieron el ingreso en 11 explotaciones por motivos sanitarios. En el caso de Carabobo se visitaron 45 granjas (58.9\%); sin embargo, 10 granjas fueron excluidas por motivos de bioseguridad, para una proporción de $77.77 \%$ (35 de 45) de explotaciones examinadas. En total se examinaron 67 granjas porcinas intensivas: 32 granjas en el estado Aragua y 35 en el estado Carabobo, de un total de 145 granjas $(46.2 \%$ del total de explotaciones de la región central de Venezuela).

En la segunda etapa del muestreo, se tomaron las muestras en cada granja de manera proporcional al total de camadas y cerdos adultos existentes en cada una ${ }^{(19)}$. Para determinar el tamaño de la muestra se empleó la fórmula descrita por Morales y Pino ${ }^{(21)}, \mathrm{n}=\mathrm{Z}^{2}$ (p). (q) / $\mathrm{EMA}^{2}$, y utilizando una prevalencia conocida de $38.9 \%{ }^{(6)}$, con $95 \%$ de nivel de confianza y un error máximo asociado (EMA) de $5 \%{ }^{(21)}$ se determinó un total de 370 muestras, sin embargo, se decidió fijar el tamaño total en 2,284 muestras, ya que se contaba con suficientes recursos y logística para la colección, envío y procesamiento de las muestras.

\section{- Toma de la muestra fecal}

En primer lugar se seleccionaron de forma aleatoria un total de 572 camadas de diferentes edades con signos de diarrea: en Aragua $(n=283)$ y Carabobo $(n=289)$. De cada camada se tomaron aleatoriamente de 4 a 5 lechones con la finalidad de realizar un "agrupamiento" de muestra en cada camada (unidad de muestreo). A cada lechón se le introdujo un hisopo por vía rectal con el propósito de estimular la defecación y colectar las heces en tubos de ensayo previamente identificados. En segundo lugar, se colectaron 1,712 muestras fecales en otros grupos de edad, estratificadas y distribuidas proporcionalmente en cada explotación de acuerdo al inventario de animales ${ }^{(19)}$. A las cerdas lactantes, gestantes, reemplazos y verracos se les tomó la muestra fecal directamente de la ampolla rectal. En grupos de lechones de iniciación, crecimiento y engorde se tomó una pequeña porción $(10 \mathrm{~g})$ recién depuesta directamente del piso en cinco puntos del corral, con la finalidad de hacer un pool de muestra en cada corral. Las muestras se estratificaron de la siguiente manera: corrales de lechones en iniciación ( $\mathrm{n}=268$ agrupamientos), corrales de lechones en crecimiento $(\mathrm{n}=172$ agrupamientos), corrales de lechones en engorde ( $\mathrm{n}=137$ agrupamientos), cerdas de reemplazo $(n=48)$, madres gestantes en edad de gestación avanzada $(n=252)$, madres lactantes de las camadas examinadas $(n=572)$ y machos reproductores $(n=263)$. En una planilla de campo se registró la edad de la camada, consistencia de las heces, número de partos de la cerda y grupos de producción, como variables asociadas a la frecuencia. Todas 
las muestras se colectaron en bolsas plásticas previamente identificadas y se introdujeron en una cava refrigerada para ser trasladadas a la Unidad de Investigación en Parasitología de la Facultad de Agronomía de la Universidad "Rómulo Gallegos", donde fueron conservadas en refrigeración hasta su procesamiento.

\section{- Análisis copro-parasitológico}

Las muestras se cultivaron a temperatura ambiente en cápsulas de Petri utilizando $20 \mathrm{ml}$ de una solución de dicromato de potasio al 2.5\% durante 24 h (agrupamiento de cada camada), y de dos semanas para el resto de grupos, de manera que exista suficiente tiempo para la esporulación de ooquistes ${ }^{(22)}$. Transcurrido ese tiempo, se empleó una técnica de centrifugación - flotación empleando una solución de azúcar - sal (1 L de solución saturada de $\mathrm{NaCl}$ más 500 g de azúcar) ${ }^{(23)}$. La visualización e identificación de ooquistes se hizo con un microscopio binocular, usando magnificación de 10 y 40X.

\section{- Análisis estadísticos 。}

Los resultados obtenidos se analizaron mediante estadísticos descriptivos y test de Jicuadrada $\left(\mathrm{X}^{2}\right)$ para determinar asociaciones estadísticas entre valores positivos con respecto a la prevalencia. Se utilizó el coeficiente con rangos de Spearman (rho) para determinar correlaciones entre prevalencia y grupos productivos, y la prueba exacta de Fisher para establecer comparaciones entre valores de prevalencia en edad de las camadas y grupos de cerdos en iniciación. El nivel de significancia para los análisis fue de 5\%. Para los cálculos se utilizó el programa estadístico Statistix ${ }^{(24)}$.

\section{Resultados y discusión}


Se encontró $C$. suis en 55 de 67 granjas examinadas $(82.1 \%)$ con niveles similares de prevalencia entre los dos estados, lo cual reflejó una alta presencia del parásito en las granjas examinadas; sin embargo, no se encontró asociación estadística $(P>0.05)$ entre los valores de prevalencia del parásito con respecto a los dos Estados: $81.3 \%$ (26/32) en el estado Aragua y $82.9 \%$ (29/35) en Carabobo. Estos resultados coinciden con lo señalado por otros autores ${ }^{(5)}$, quienes encontraron $93.3 \%$ de prevalencia en granjas de la región centro - occidental de Venezuela, mientras $75 \%$ en granjas de la región central ${ }^{(6,11)}$. Al referirlo a los lechones, se determinó $36.7 \%$ de prevalencia (210/572). Estos resultados coinciden con lo señalado en otros estudios $^{(5,6,11)}$, donde determinaron valores de prevalencia similares en lechones lactantes, y podría deberse a la falta de programas de higiene y desinfección de las unidades de parición. Sin embargo, estos resultados difieren con lo señalado por Driesen et $a l^{(7)}$ y Otten et $a l^{(8)}$, quienes señalaron mayores valores de prevalencia en camadas.

En el Cuadro 1 se muestra la comparación entre los valores de frecuencia de Cystoisospora suis por grupo de edad de las camadas muestreadas. De acuerdo a estos resultados, se encontraron diferencias entre los cuatro grupos examinados $(P<0.05)$, mostrando mayor frecuencia en camadas de 1 a 7 y 8 a 14 días de edad. Estos resultados coinciden con lo reportado en otras investigaciones ${ }^{(6,10)}$, donde señalaron mayores valores de prevalencia en camadas de dos semanas de edad. Posiblemente, la contaminación del paritorio en estas granjas provoca una elevada presión de infección, lo que trae como consecuencia que los animales se infecten en sus primeros días de vida. Sin embargo, existen evidencias que indican mayores tasas de frecuencia en lechones de tres y cuatro semanas de $\operatorname{edad}^{(8,9)}$.

Cuadro 1: Frecuencia de Cystoisospora suis por grupo de edad de las camadas muestreadas

\begin{tabular}{cccc}
\hline Edad (días) & Positivas & Total & Frecuencia (\%) \\
\hline 1 a 7 & 55 & 138 & $9.6^{\mathrm{a}}$ \\
8 a 14 & 107 & 249 & $18.7^{\mathrm{a}}$ \\
15 a 21 & 32 & 138 & $5.5^{\mathrm{b}}$ \\
> 21 & 16 & 47 & $2.9^{\mathrm{b}}$ \\
Total & 210 & 572 & 36.7 \\
\hline
\end{tabular}

ab Valores con diferente superíndice difieren significativamente $(P<0.05)$.

En la Figura 1 se muestra la distribución de frecuencia de muestras positivas en camadas con menos de 7 días de vida. De un total de 55 muestras positivas, nueve muestras pertenecían a camadas menores a tres días de edad, para una frecuencia acumulada de $16.3 \%$. Igualmente, se determinaron seis camadas $(10.9 \%)$ con menos de $24 \mathrm{~h}$ de nacidas excretando ooquistes, lo que representa un resultado importante en el comportamiento epidemiológico del parásito. El período prepatente de $C$. suis es de cuatro días ${ }^{(1)}$, por lo tanto, la presencia de ooquistes en 
camadas menores a tres días de edad podría deberse a varias razones. En primer lugar, el cultivo fecal en solución de dicromato de potasio confirma la presencia de ooquistes de $C$. suis, ya que la esporulación (100\%) permite diferenciar con respecto a ooquistes de Eimeria spp. En segundo lugar, se podría pensar en la presencia de ooquistes en tránsito intestinal, o bien, que estos lechones recién nacidos se hayan contaminado con heces de camadas previas, de la madre, o de las tetas de la cerda. Sin embargo, esta hipótesis se descarta, ya que tendrían que haber esporulado para poder resistir la temperatura y humedad del paritorio, además, en el caso que la contaminación fuese del ambiente, ooquistes no esporulados en tránsito intestinal no serían capaces de sobrevivir debido a la acción de ácidos y enzimas digestivas. Otra respuesta a este hallazgo sería la existencia de alguna ruta alternativa de infección, probablemente por la existencia de formas evolutivas extra - intestinales, en el que cerdas madres puedan transferir formas evolutivas del protozoario a los lechones, sin embargo, esta hipótesis aún está en discusión ${ }^{(25,26)}$.

Figura 1: Distribución de frecuencia de muestras positivas a Cystoisospora suis en los primeros 7 días de vida

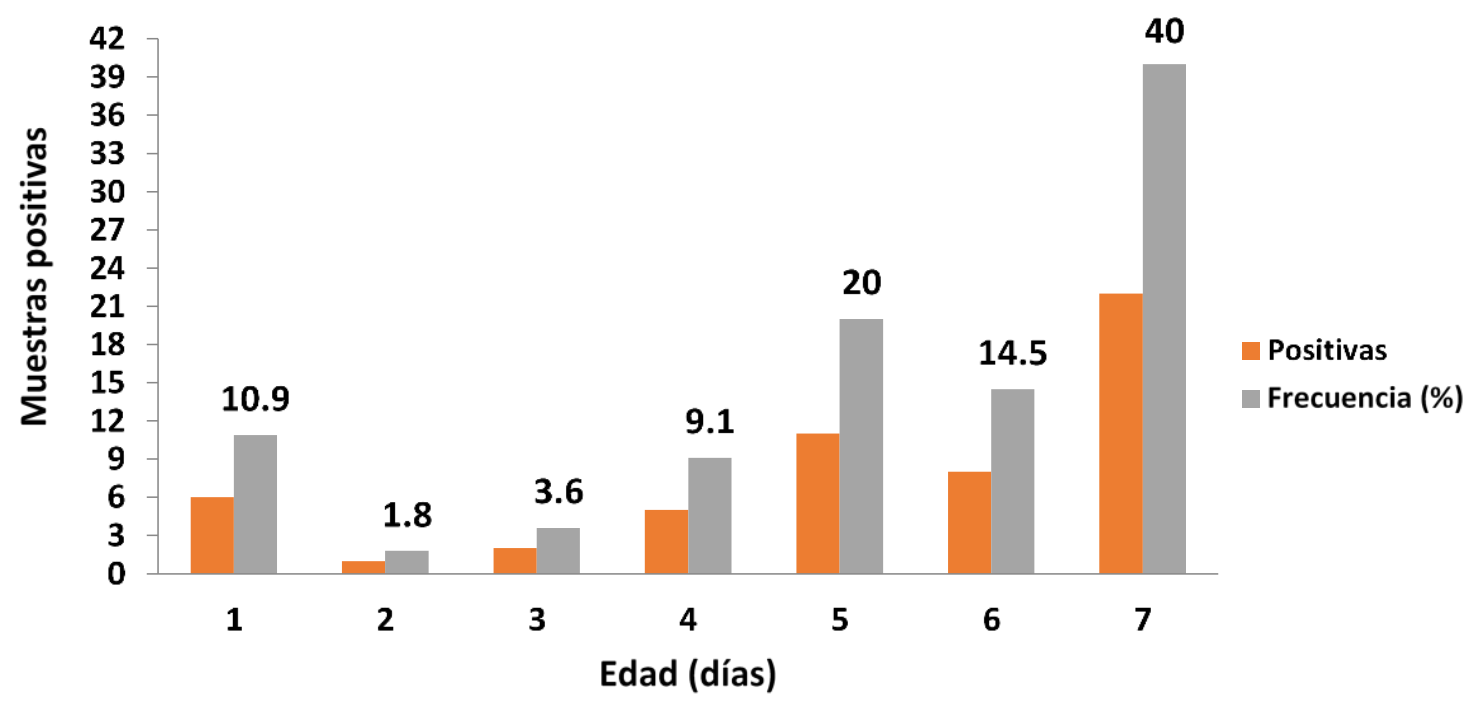

Algunos investigadores han señalado la posibilidad de la existencia de formas evolutivas en hígado y tejidos linfoides (bazo y ganglios), tomando en consideración la presencia de brotes

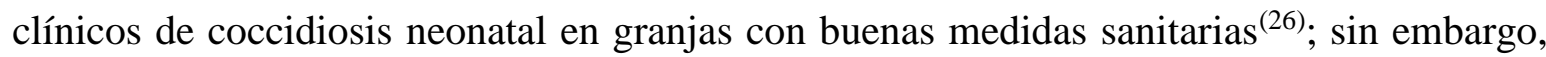
existen estudios que no comprobaron la existencia de formas evolutivas extra - intestinales en lechones y ratones inoculados experimentalmente con $C$. suis ${ }^{(1)}$. La presencia de formas evolutivas extra - intestinales ha sido comprobada para especies de Cystoisospora en caninos y felinos ${ }^{(1,27)}$, así como $C$. belli en humanos ${ }^{(1)}$. Probablemente merozoítos, en vez de continuar con su ciclo normal de desarrollo en el tracto intestinal, dejan el intestino y se 
diseminan por vía linfática para invadir linfonódulos mesentéricos, así como otros órganos del sistema retículo endotelial ${ }^{(1)}$, y especialmente en pacientes inmuno - suprimidos. Por otra parte, la falta de cuerpo de Stieda en los esporoquistes de C. suis sugieren la ocurrencia de formas extra - intestinales en el ciclo de vida del protozoario, tal y como sucede con el grupo de Cystoisosporas que afectan a perros y gatos ${ }^{(27)}$. Se podría pensar que cerdas gestantes mantenidas en granjas con pobres condiciones sanitarias y estados de inmuno - supresión ocasionados por efecto del estrés, así como por infecciones como el síndrome respiratorio y reproductivo porcino (PRRS) y circovirus porcino tipo 2 (CPV tipo II), albergan formas evolutivas de $C$. suis en tejidos extra - intestinales, y de esta manera viajar por vía linfo hematógena (transplacentaria) e infectar a sus fetos. En el momento del nacimiento, los lechones se infectan por vía transplacentaria y actuarían como agentes multiplicadores y diseminadores del parásito al resto de las camadas.

En la Figura 2 se observa la dinámica de excreción de ooquistes en camadas, y se muestra la formación de una curva trifásica con tres picos de excreción: un primer pico a los 7 días de edad, un segundo y tercer pico de excreción a los 12 y 21 días, respectivamente. Estos resultados difieren con lo señalado por algunos autores, quienes señalaron que en lechones inoculados experimentalmente el ciclo de desarrollo de $C$. suis es bifásico, con la formación de dos picos de excreción e interrupción en la excreción durante dos o tres días entre el primero y segundo período de eliminación ${ }^{(1,28)}$. La presencia de un segundo pico de excreción de ooquistes es causada por un segundo ciclo interno de desarrollo del protozoario (segunda esquizogonia); sin embargo, hay autores que postularon que este segundo pico se debe al retorno de formas extra - intestinales hasta el tracto intestinal, hipótesis que aún no ha sido comprobada $^{(28)}$. Existen reportes que demuestran un tercer pico de excreción de ooquistes en lechones experimentalmente infectados ${ }^{(29)}$. El tercer pico de excreción refleja un retraso en la resistencia inmunológica contra el parásito, resultado de una pobre estimulación antigénica, e incluso, esto podría explicar el retorno en la excreción de ooquistes en cerdos de 5 a 6 semanas de edad cuando son trasladados a otra unidad de producción o corral, que teóricamente se ven afectados por el estrés causado por el destete. 
Figura 2: Dinámica de excreción de ooquistes de Cystoisospora suis en las camadas muestreadas

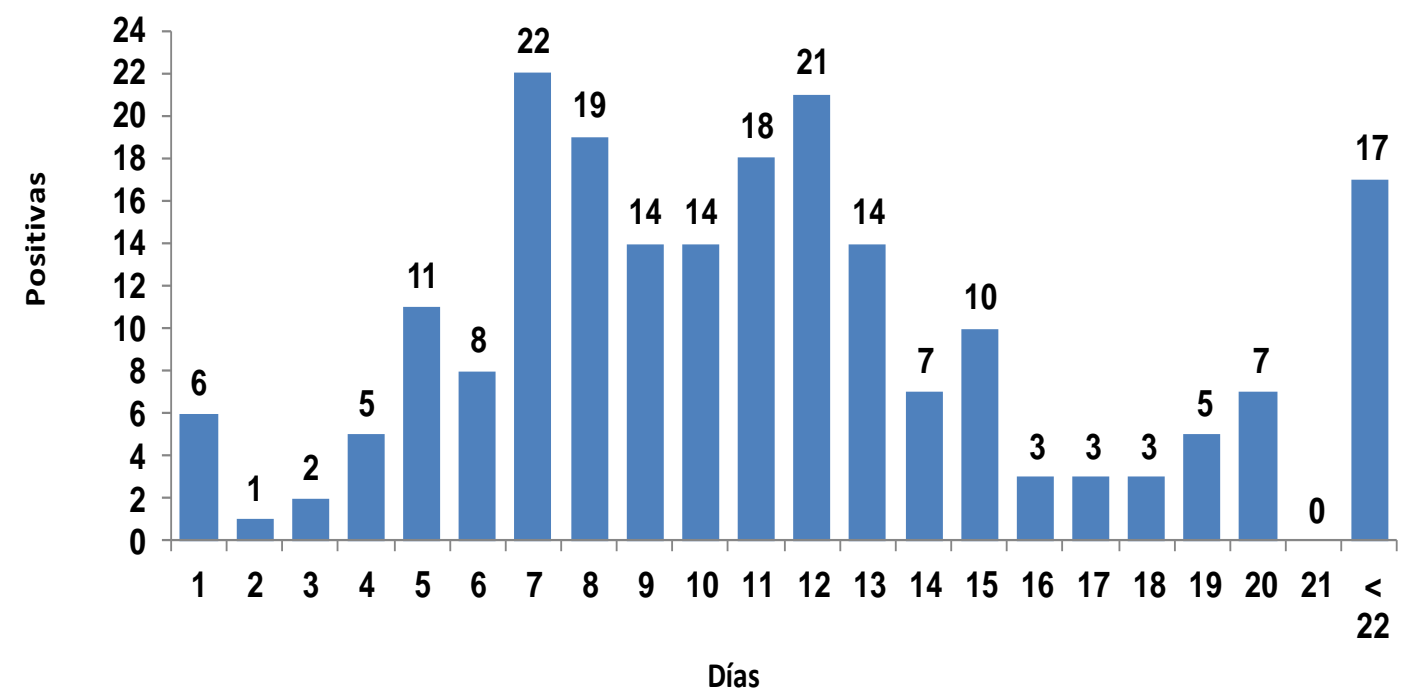

En la Figura 3 se muestra la frecuencia a $C$. suis en camadas provenientes de cerdas con diferente número de partos. Los resultados obtenidos se analizaron mediante una prueba de correlación con rangos de Spearman, indicando que a mayor número de partos, menor es la frecuencia en camadas $(\mathrm{rho}=-0.77 ; P<0.05)$. El efecto del número de partos sobre la frecuencia de $C$. suis ha sido señalado en otros estudios ${ }^{(6)}$; sin embargo, no se demostraron diferencias estadísticas entre valores de frecuencia a $C$. suis en camadas provenientes de cerdas con diferente número de partos $^{(3)}$. El número de partos de la cerda parece ser un factor de riesgo importante que puede afectar el desempeño productivo en las salas de parto, y por eso en muchas empresas porcinas se busca mantener cierta uniformidad en esta variable. Se ha señalado que las cerdas adquieren mejor status inmunitario en la medida que se hacen viejas y esto ha sido comprobado para Micoplasma hyopneumoniae ${ }^{(12)}$; así mismo, se han comprobado diferencias estadísticas respecto a infecciones por Haemophilus parasuis en cerdas con diferente número de partos ${ }^{(13)}$. Probablemente, los lechones que se crían con cerdas primerizas no reciben la cantidad y calidad de anticuerpos calostrales para combatir infecciones por $C$. suis, mientras que aquéllos que se crían con cerdas adultas con sistemas inmunológicos más desarrollados, reciben anticuerpos necesarios que permiten al lechón adquirir una mejor inmunidad pasiva y combatir infecciones por $C$. suis. 
Figura 3: Valores de frecuencia a $C$. suis en camadas provenientes de cerdas con diferente número de partos

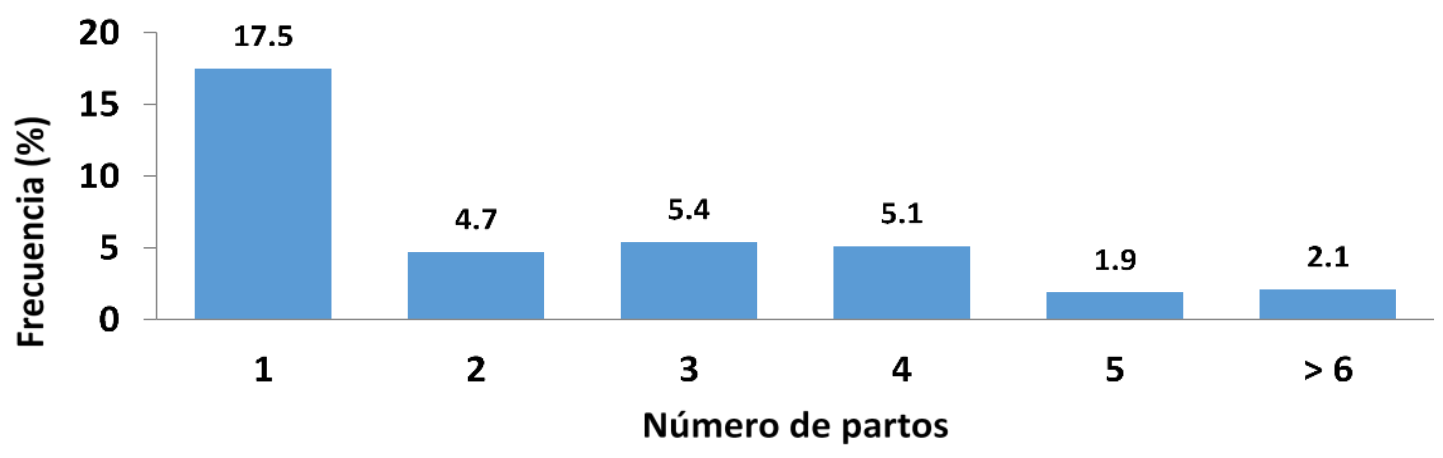

Correlación con rangos de Spearman $(\mathrm{rho}=-0.77 ; P<0.05)$.

Los lechones neonatos tienen un sistema inmune inmaduro, y la transferencia calostral de células inmunes y anticuerpos es esencial para controlar las infecciones a esa edad; sin embargo, se ha demostrado que los anticuerpos calostrales no protegen contra la enfermedad $^{(30,31,32)}$. Algunos autores demostraron la transferencia de células inmunes y anticuerpos específicos contra $C$. suis a través del calostro, pero estos anticuerpos no proporcionaron protección contra la manifestación clínica de la enfermedad ${ }^{(30,31,32)}$. Por otro lado, la inmunidad mediada por células juega un rol muy importante en la resistencia a la enfermedad en los lechones ${ }^{(33)}$; sin embargo, cerdas primerizas con bajo estatus inmunológico no son capaces de transferir a sus hijos los elementos necesarios para el desarrollo de mecanismos de respuesta no específicos (activación de complemento, fagocitosis y citoquinas), lo que significa, que los lechones viven sus primeras semanas de vida con un sistema inmune vulnerable, y por tanto no son capaces de contrarrestar la infección.

En cerdas primíparas, la falta de una continua estimulación antigénica pudiera ser un factor que hace vulnerable a sus crías contra la infección por $C$. suis, a diferencia de cerdas multíparas que han estado en mayor contacto con el protozoario, y por tanto han desarrollado un sistema inmunológico capaz de inducir una mejor respuesta en lechones lactantes. Probablemente, la respuesta inmune ante infecciones primarias (inmunidad innata) en lechones de cerdas primíparas sea muy baja; sin embargo, en la medida que el lechón va creciendo, se activan los mecanismos de inmunidad adaptativa capaces de contrarrestar la infección. 
Con respecto a la presencia del protozoario según la consistencia de las muestras, se encontraron diferentes grados de positividad, lo que refleja que hubo asociación estadística $(P<0.05)$ con respecto a la consistencia. Aunque hubo excreción de ooquistes en los tres tipos de consistencia, se observó una frecuencia de $64.8 \%$ en heces de consistencia semipastosa y pastosa. Estos resultados coinciden con lo señalado por otros autores, quienes indicaron mayor frecuencia de $C$. suis en muestras de consistencia cremosa, que aquellas líquidas ${ }^{(3,6)}$. Existen estudios que indican mayor frecuencia del parásito en muestras acuosas (35.2\%), y menor frecuencia en muestras de consistencia pastosa ${ }^{(34)}$. La diarrea en lechones se explica, por la acción patógena que causa el parásito en el epitelio intestinal en el transcurso de las diferentes etapas que se cumplen en la fase endógena del ciclo de vida. Durante la esquizogonia se produce diarrea debido a la invasión y multiplicación del parásito dentro de la célula epitelial, sin embargo, la producción de ooquistes es muy baja. En la medida que avanza la infección la diarrea va cediendo, y la consistencia del excremento comienza a hacerse pastosa, e incluso normal, hasta que se cumpla el ciclo de vida del parásito con la debida producción de ooquistes. De acuerdo a esto, es improbable conseguir animales excretando ooquistes y con producción de heces liquidas, por lo que habría que considerar otros copatógenos como E. coli, Rotavirus y Cryptosporidium.

Con respecto a los resultados en cerdos adultos, se determinó que todos los grupos evaluados presentaron excreción de ooquistes, con excepción de las cerdas de reemplazo (Cuadro 2). Los resultados obtenidos se analizaron mediante una prueba de correlación con rangos de Spearman, el cual no arrojó correlación estadística ( rho $=0.03 ; P>0.05)$, lo que indica que la edad y la frecuencia a $C$. suis en cerdos adultos actúan de manera independiente. Las cerdas lactantes y machos reproductores mostraron 9.4 y $4.9 \%$ de frecuencia a $C$. suis, respectivamente, con valores muy similares entre los dos estados. Los hallazgos en cerdas madres difieren con lo señalado por otros autores, quienes no encontraron la infección en cerdas madres, por tanto, concluyen que las cerdas no juegan ningún rol en la cadena de transmisión del parásito ${ }^{(1,2)}$. Sin embargo, existen evidencias de infección en cerdas madres $^{(3,4,5)}$. La baja presencia de ooquistes de $C$. suis en excremento de madres y verracos, demuestra que estos grupos pueden excretar cantidades de ooquistes que no son detectables con las pruebas convencionales; sin embargo, esas cantidades bajas de ooquistes pueden tornarse infectivas y diseminarse por toda la paridera, sobre todo cuando existen problemas higiénico - sanitarios y de manejo. Por tal motivo, las cerdas podrían jugar un papel importante en la cadena de transmisión, bien sea por un parasitismo activo no determinado, o por transporte pasivo de ooquistes a sus lechones. Con relación a cerdos después del destete, se determinó excreción de ooquistes en las tres etapas, con especial significancia en iniciación $(23.8 \%)$. Posiblemente, lechones destetados y mantenidos en corrales próximos al área de maternidad pueden actuar como fuentes diseminadoras del parásito, sobre todo en aquellas granjas donde no aplican programas de higiene y desinfección de instalaciones. 
Cuadro 2: Frecuencia de Cystoisospora suis en grupos de cerdos adultos de la región central de Venezuela

\begin{tabular}{lccc}
\hline Grupos & Aragua & Carabobo & Total \\
\hline Iniciación & 25.1 & 22.5 & 23.8 \\
Crecimiento & 2.2 & 4.7 & 3.4 \\
Engorde & 3.3 & 3.8 & 3.6 \\
Reemplazos & 0 & 0 & 0 \\
Gestantes & 4.1 & 6 & 5.1 \\
Lactantes & 8.8 & 10 & 9.4 \\
Verracos & 4.6 & 5.1 & 4.9 \\
\hline
\end{tabular}

Coeficiente de correlación con rangos de Spearman (rho= 0.03), $P>0.05$.

$\mathrm{Al}$ aumentar el número de partos, disminuye la frecuencia en madres (Cuadro 3). Según estos resultados existe asociación estadística, y se observa mayor infección en cerdas primerizas, probablemente debido a factores inmunológicos involucrados. Probablemente, las cerdas durante su crecimiento mantienen y esconden formas evolutivas intra y extra - intestinales del protozoario ${ }^{(34)}$, y cuando llega el primer parto comienzan a excretar cantidades suficientes de ooquistes que son detectados por medio de técnicas de concentración - flotación; sin embargo, en la medida que avanzan en edad, sus sistemas inmunológicos se desarrollan hasta el punto de contrarrestar la infección y por tal motivo disminuye la cantidad de ooquistes, los cuales no son detectables por técnicas convencionales de flotación. También se podría pensar que cerdas primerizas se tornan más vulnerables que cerdas viejas a los cambios ocurridos en el traslado a la sala de partos, ruidos, personal y eventos del primer parto, ocasionando un gran estrés a estos animales que pudieran alterar su sistema inmunológico y de esta manera iniciar la excreción de ooquistes de C. suis, y actuar como posibles fuentes de infección. Se ha señalado que los nuevos eventos y cambios que ocurren en el parto de las cerdas (dolor, nerviosismo) son fuente principal de estrés, sobre todo en cerdas primíparas, las cuales también se pueden ver inmunocomprometidas por efecto de la lactancia ${ }^{(13,14,15)}$. 
Cuadro 3: Frecuencia a Cystoisospora suis en cerdas lactantes de acuerdo al número de partos

\begin{tabular}{cccc}
\hline Partos & Positivas & Total & Frecuencia $(\%)$ \\
\hline 1 & 37 & 173 & 6.5 \\
2 & 7 & 106 & 1.2 \\
3 & 5 & 117 & 0.9 \\
4 & 4 & 83 & 0.7 \\
5 & 1 & 44 & 0.2 \\
$>6$ & 0 & 49 & 0 \\
Total & 54 & 572 & 9.4 \\
\hline
\end{tabular}

Coeficiente de correlación con rangos de Spearman (rho= - 0.98).

En el Cuadro 4 se muestra la comparación entre valores de frecuencia a $C$. suis en lechones de diferentes semanas de producción. Según estos resultados existen diferencias $(P<0,05)$ en los seis grupos examinados, los animales con cuatro y cinco semanas de edad mostraron mayores valores de infección (frecuencia acumulada de $16 \%$ ). El destete de los cerdos ha sido señalado como el momento de mayor estrés en la etapa productiva del animal, y el estrés ocasionado por el destete se ha asociado con la excreción de ooquistes de C. suis en cerdos de cinco y seis semanas de edad ${ }^{(35,36)}$. Indudablemente, la respuesta inmune del lechón ante cualquier agente patógeno puede verse comprometida por situaciones de manejo que desencadenen estrés. Existen suficientes evidencias que señalan que el estrés tiene efectos sobre el sistema inmune del cerdo. En tal sentido, se ha comprobado que el estrés aumenta los niveles sanguíneos de corticosteroides, especialmente cortisol, pudiendo reducir la proliferación de linfocitos, así como el tamaño de los linfonódulos y, por consiguiente, disminución del número de anticuerpos producidos ${ }^{(35,36)}$. Esta alteración en los mecanismos inmunes del lechón podría jugar un papel importante en la aparición de ooquistes en cerdos jóvenes; sin embargo, se desconoce el papel que juegan las cerdas madres en la respuesta inmune del lechón. 
Cuadro 4: Frecuencia a Cystoisospora suis en cerdos de iniciación de diferentes semanas de edad

\begin{tabular}{cccc}
\hline Semana & Positivos & Total & Frecuencia $(\%)$ \\
\hline 4 & 21 & 63 & $7.8^{\mathbf{a}}$ \\
5 & 22 & 69 & $8.2^{\mathbf{a}}$ \\
6 & 9 & 62 & $3.4^{\mathbf{b}}$ \\
7 & 3 & 32 & $1.1^{\mathbf{c}}$ \\
8 & 7 & 34 & $2.6^{\mathbf{b}}$ \\
9 & 2 & 8 & $0.7^{\mathbf{c}}$ \\
Total & 64 & 268 & 23.9 \\
\hline
\end{tabular}

${ }^{\text {ab }}$ Valores con diferente superíndice difieren significativamente $(P<0.05)$.

\section{Conclusiones e implicaciones}

Existe una amplia distribución y frecuencia del protozoario en granjas porcinas ubicadas en la región central de Venezuela, que pudieran ser controladas al mejorarse las condiciones higiénico - sanitarias de las explotaciones. Se determinó infección en cerdas madres y lechones en iniciación, lo que supone que estos grupos podrían actuar como fuentes de infección y diseminación del parásito. La presencia de ooquistes en muestras de camadas menores a tres días de edad, representa un hallazgo importante en el comportamiento epidemiológico del parásito, que presupone la existencia de alguna ruta alternativa de la infección. El número de partos de la cerda tuvo impacto sobre la frecuencia del protozoario en la población muestreada. Probablemente estos hallazgos se asocian con mecanismos inmunológicos desconocidos.

\section{Agradecimientos}


Al Programa de Medicina Veterinaria de la Facultad de Ciencias Agropecuarias de la Universidad de Santander por su valiosa colaboración y apoyo en la ejecución de este trabajo.

\section{- Literatura citada}

1. Lindsay D, Dubey J. Coccidia and other protozoa. In: Straw BE, et al editors. Diseases of swine. $9^{\text {th }}$ ed. Ames, Iowa, USA: Iowa State University; 2005:861-873.

2. Farkas R, Szeidemann Z, Majoros G. Prevalence and geographical distribution of Isosporosis in swine farms of Hungary [abstract]. In: Proc 18th IPVS Congress, Hamburg, Germany. 2004:314.

3. Meyer C, Joachim A, Daugschies A. Ocurrence of Isospora suis in larger piglet production units and on specialized piglet rearing farms. Vet Parasitol 1999;82:277284.

4. Karamon J, Ziomko I, Cencek T. Prevalence of Isospora suis and Eimeria spp. in suckling piglets and sows in Poland. Vet Parasitol 2007;147:171-175.

5. Pinilla J, Coronado A. Prevalencia de Isospora suis en lechones criados en granjas de la región Centro - Occidental de Venezuela. Zoo Trop 2008;26(1):47-53.

6. Pinilla J. Prevalencia de Isospora suis en granjas porcinas intensivas ubicadas en el estado Aragua, Venezuela. Zoot Trop 2009;27(2):1-9.

7. Driesen SJ, Carland PG, Fahy VA. Studies on preweaning piglet diarrhoea. Aust Vet J 1993;70:259263.

8. Otten A, Takla M, Daugshies A, Rommel M. The epizootiology and pathogenic significance of infections with Isospora suis in ten piglet production operations in Nordrhein-Westfalen. Berl Mun Tier Woch 1996;109(6-7):220-223.

9. Niestrath M, Takla M, Joachim A, Daugschies A. The role of Isospora suis as a pathogen in conventional piglet production in Germany. J Vet Med B 2002;49:176-180.

10. Hamadejova K, Vitovec J. Ocurrence of the coccidium Isospora suis in piglets. Vet Med Czech 2005;50(4):159-163. 
11. González Y de W, De Moreno L, García G. Isospora suis en granjas con diferentes condiciones de instalaciones y manejo. Vet Trop 2000;25(2):257-265.

12. Cardona A, Pijoan C, Utrera V, Deen J. Prevalence of Mycoplasma hyopneumoniae in different parity cull sows [abstract]. In: Proc 18th IPVS Congress, Hamburg, Germany. 2002:402.

13. Holyoake PK. Dam parity affects the performance of nursery pigs [abstract]. In: Proc 19th IPVS Congress, Copenhagen, Denmark. 2006:149.

14. Klopfstein C, Farmer C, Martineau G. Diseases of the mammary glands and lactation problems. In: Straw BE, et al. editors. Diseases of swine. 8th ed. Ames, Iowa, USA: Iowa State University Press;1999:833-860.

15. Lafranchi E, González J, Filios S. The efficacy of butafosfan in the periparturient sow Field trial Mexico [abstract]. In: Proc 20th IPVS Congress, Durban, South Africa. 2008:479.

16. Feporcina. Comportamiento del sector porcino en el 2005. Revista de Información Divulgativa. 2010;1:10-12.

17. Ministerio del Ambiente y Recursos Naturales Renovables (MARNR). Anuario climatológico. MARNR. Estados Aragua y Carabobo. 2015.

18. Rodríguez D. Estudio Seroepidemiológico de la enfermedad de Aujeszky en granjas porcinas del estado Carabobo [tesis maestría]. Maracay. Universidad Central de Venezuela; 1995.

19. Lohr S. Muestreo: Diseño y análisis. México: International Thomson Editores; 2000.

20. SASA - Aragua y Carabobo. Censo de granjas porcinas de los estados Aragua y Carabobo. En: Reportes de programa vacunación contra Fiebre Aftosa. Departamento de epidemiología. Servicio Autónomo de Sanidad Agropecuaria; 2015.

21. Morales G, Pino A. Parasitología cuantitativa. Fundación Fondo Editorial Acta Científica Venezolana, Caracas. 1987.

22. Hendrix CM. Diagnóstico parasitológico veterinario. 2da ed. Madrid, España: Editorial Harcourt Brace; 1999.

23. Henriksen S, Christensen J. Demonstration of Isospora suis oocysts in faecal samples. Vet Rec 1992;131:443-444.

24. Statistix 8. Analytical Software for Windows. USA. 2008. 
25. Sotiraki S, Roepstorff A, Nielsen J, Maddox - Hyttel C, Enoe C, Boes J, Murrell K, Thamsborg S. Population dynamics and intra-litter transmissions patterns of Isospora suis in suckling piglets under on- farms conditions. Parasitol 2008;135(3):395-405.

26. Cordero del Campillo M, Hidalgo M, Díez N. Parasitosis del cerdo. Eimeriosis e Isosporosis. En: Cordero del Campillo et al. editores. Parasitología veterinaria. Madrid, España: Editorial McGraw-Hill. Interamericana; 1999:451-456.

27. Dubey J, Frenkel J. Extra - intestinal stages of Isospora felis and I. rivolta (Protozoa: Eimeriidae) in cats. J Protozool 1972;19:89-92.

28. Harleman J, Meyer R. Life cycle of Isospora suis in gnotobiotic and conventional piglets. Vet Parasitol 1984;17:27-39.

29. Christensen J, Henriksen S. Shedding of oocysts in piglets experimentally infected with Isospora suis. Act Vet Scand 1994;35:165-172.

30. Schwarz L, Worliczek H, Winkler M, Joachim A. Superinfection of sows with Cystoisospora suis ante partum leads to a milder course of cystoisosporosis in suckling piglets. Vet Parasitol 2014;204(3-4):58-168.

31. Schwarz L, Joachim A, Worliczek H. Transfer of Cystoisospora suis-specific colostral antibodies and their correlation with the course of neonatal porcine cystoisosporosis. Vet Parasitol 2013;197(3-4):487-497.

32. Shrestha A, Abd-Elfattah A, Freudenschuss B, Hinney B, Palmieri N, Ruttkowski B, Joachim A. Cystoisospora suis - A model of mammalian cystoisosporosis. Front Vet Sci 2015;2:68. Doi: 10.3389/fvets.2015.00068.

33. Koudela B, Kucerová S. Inmunity against Isospora suis in nursing piglets. Parasitol Res 2000;86:861-863.

34. Pavlovic I, Savic B, Jakic - Dimic D. Prevalence of coccidiosis in farm - farrowing conditions [abstract]. In: Proc 20th IPVS Congress, Durban, South Africa. 2008:345.

35. Nillson O. Isospora suis in pigs with post weaning diarrhea. Vet Rec 1988;122:310-311.

36. Roth JA. The system immune. In: Straw BE, et al editors. Diseases of swine. 8th ed. Ames, Iowa, USA: Iowa State University Press; 1999:799-820. 